\title{
LA HISTORIA BRITANNIE \\ DE GEOFFREY DE MONMOUTH \\ EN TRES VERSIONES HISPÁNICAS
}

\author{
Antonio Contreras Martín \\ Institut d'Estudis Medievals \\ tcontreras@telefonica.net
}

La Historia Regum Britanniae (hacia II36) ${ }^{\mathrm{x}}$, de la que se tuvo un amplio conocimiento en las tierras británicas, se difundió en otros ámbitos territoriales y lingüísticos europeos (Echard, 20II), ya en época temprana (Breta Sögur en islandés, finales del siglo XII) (Gropper, 2OII). La obra de Geoffrey de Monmouth circuló de forma independiente o integrada, generalmente de forma fragmentaria, en las llamadas 'crónicas universales' (por ejemplo, Spiegel historiel del flamenco Jacob van Maerlant, hacia I285) (Besamusca, 2000, pp. I88-190).

En la Península Ibérica, han pervivido, al menos hasta la fecha, tres versiones parciales: una en castellano, otra en catalán y la tercera en aragonés.

En el reino castellano-leonés, el testimonio conservado forma parte de la General Estoria ${ }^{2}$, ambiciosa obra inacabada, 'proyecto de crónica universal', patrocinada por Alfonso X el Sabio en el último tercio del siglo XIII (Kasten, I970 y Rico, 1984).

Por su parte, en los territorios de la Corona de Aragón, han pervivido dos versiones: una en catalán, conservada en el Ms Esp. I3 de la BNF (París) (fols. 83$97^{v}$ ), copia del siglo Xv de un original datable en la primera mitad del siglo xIV, que es también un caso de 'proyecto de crónica universal'; y otra, en la variedad romance aragonesa, integrada en La Grant Crónica de Espanya de Juan Fernández de Heredia (antes de I385) (Ms. IoI33 de la BNE, Madrid, fols. 6I $\mathrm{I}^{\mathrm{r}}-7 \mathrm{I}^{\mathrm{v}}$ ) (Cacho Blecua, 1997), que podría derivar del texto catalán según Bohigas (1985, p. 179), pero que por su concepción y configuración es, sin duda, deudora de la obra alfonsít.

I Faral (1993), Wright (1988 y 1985), A partir de ahora $H R B, F V$ y BernM, respectivamente. Para los antropónimos y topónimos, sigo las formas propuestas por L. A. de Cuenca en Geoffrey de Monmouth (1984).

2 Alfonso X el Sabio (2009). Desde aquí $G E$.

3 Bohigas (1985). A partir de aquí $H R B C$.

4 Fernández de Heredia. Desde ahora GCE.

MEdievalia i6 (2013), 29-35

ISSN: O2II-3473 (paper), 20I4-84IO (digital) 
Ante todo, es necesario destacar que, mientras que tanto la versión catalana como la de Fernández de Heredia ofrecen un relato lineal y consecutivo, que se desarrolla como un bloque unitario, la obra del taller alfonsí imbrica la narración de los personajes britones con la historia del pueblo judío y con la de los griegos, romanos y germanos, en su afán de confeccionar una crónica global ('general'), que diese cuenta de la historia simultánea de todos los pueblos.

La narración contenida en $G E$ abarca desde 'el rey Eneas Silvio, padre de Bruto hasta 'la guerra de Cunedagio y Margano' (Alfonso X el Sabio, 2009, II/2, pp. 495-525 y 6I5-6I6, y III/I, pp. 303-315, 479-480, 499 y 570-576), la de $H R B C$ se inicia con la 'Descripción de Britania' y se extiende hasta 'el rey Dunvalón Molmucio' (Bohigas, 1985, pp.I78-203), y la GCE sólo recoge la 'historia de Bruto' (fols. $6 \mathrm{I}^{\mathrm{r}}-7 \mathrm{I}^{\mathrm{v}}$ ), que se corresponden respectivamente con $H R B, F V$ y BernM (caps. 6-32, 5-34 y 6-22):

\begin{tabular}{|c|c|c|c|}
\hline HRB & GE & HRBC & GCE \\
\hline $\begin{array}{l}\text { Descripción de Brita- } \\
\text { nia (cap. I) }\end{array}$ & & (pp. I8O-I8I) & \\
\hline $\begin{array}{l}\text { Historia de Bruto y } \\
\text { su padre Eneas Silvio } \\
\text { (caps. 6-22) }\end{array}$ & $\begin{array}{l}\text { (II/2, pp. 495-525, } \\
6 \mathrm{I} 6 ; \mathrm{III} / \mathrm{I}, \mathrm{pp} .303- \\
308)\end{array}$ & (pp. I8I-I94) & (fols. $6 \mathrm{I}^{\mathrm{r}}-7 \mathrm{I}^{\mathrm{v}}$ ) \\
\hline Locrino (caps. 23-25) & (III/I, pp. 303-3I2) & (pp. 194-196) & \\
\hline Güendolena (cap. 25) & (III/I, pp. 3I2-3I3) & (p. 196) & \\
\hline Mempricio (cap. 26) & $(\mathrm{III} / \mathrm{I}, \mathrm{pp} \cdot 3 \mathrm{I3}-3 \mathrm{I} 4)$ & (p. 196) & \\
\hline Ebrauco (cap. 27) & (III/I, pp. 3I4-3I5) & (pp. 196-197) & \\
\hline $\begin{array}{l}\text { Bruto el del Verde } \\
\text { Escudo (cap. } 28 \text { ) }\end{array}$ & (III/I, p. 479) & (p. 197) & \\
\hline Leil (cap. 28) & (III/I, p. 479) & (p. 197) & \\
\hline $\begin{array}{l}\text { Rud Hudibrás (cap. } \\
\text { 29) }\end{array}$ & $\begin{array}{l}\text { (III/I, pp. 479-48o, } \\
499)\end{array}$ & (p. 197) & \\
\hline Bladud (cap. 30) & (III/I, pp. 570-57I) & (p. 198) & \\
\hline El rey Lear (cap. 3I) & (III/I, pp. 57I-574) & (pp. I98-20I) & \\
\hline 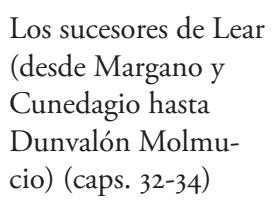 & (III/I, pp. 574-576) & (pp. 20I-203) & \\
\hline
\end{tabular}


El cotejo de las tres versiones con el original, tanto con el texto 'canónico' $(H R B)$ como con sus variantes ( $F V$ y BernM), revela que mantienen un alto grado de fidelidad:

...ita ut a regibus et principibus prae omni juventute patriae amaretur. Erat enim inter sapientes sapiens, inter bellicosos belicosus et, quicquid aure vel argenti sive ornamentorum acquirebat, totum militibus erogabat. Divulgata itaque per universas nationes ipsius fama (HRB, cap. 7).

...que era amado de los reyes e de los príncipes más que ningún otro de esa tierra, ca era Bruto de buenas costumbres e era sabio entre los sabios e lidiador entre los lidiadores, e quequier que ganava, oro o plata o otras cosas preciadas, dávalo todo a los cavalleros, onde se apublicó la fama e la prez d'este Bruto por todas las gentes d'aquella tierra ( $G E$, II/2, p. 498).

...que era amat e per reys e per princeps sobre tots los joves de la terra; car ell era entre los savis, fort savi; entre homes d'armes usantz e guerras, valent e ardit e molt tractant, e quant conqueria d'aur e d'argent, tot ho donava als cavallers, axi que res ab si no retenia. Divulgada la sua fama per totes nacions (HRBC, p. 182).

...que era amado e por reyes e por prínçipes sobre todos los jóvenes de la tierra, que él <el> era entre los savios muyt savio, entre los hombres de guerra e usantes armas era ardit e muyt tractant, e todo cuanto guenava $<\mathrm{n}>$ de oro e de argent todo lo dava a los cavalleros, assí que res no se aturava, divulgada o sabida la su fama por todas las naciones $\left(G C E \text {, fol. } 6 \mathrm{r}^{\mathrm{va}}\right)^{\mathrm{s}}$.

A pesar de ello, presentan algunas divergencias, consistentes en abreviaciones o supresiones (reductio):

Concordia tamen habita, retinuit eum alter generorum, Maglaunus, dux Albaniae, cum XL militibus ${ }^{6}$, ne secum inglorius maneret. Elapso deinde biennio, moram eo apud generum faciente, indignata est Gonorilla, filia sua, ob multitudinem militum ejus, qui convicia ministris inferebant, qui sibi profusior epimenia non praebebatur. Proinde, maritum suum affata, jussit patrem obsequio $\mathrm{xx}$ militum contentum esse $\mathrm{e}^{7}$, relictis ceteris quos habuerat $(H R B \text {, cap. 3I })^{8}$.

E feta pau e concordia, si Leir stigues per si mateix no li fora honor, la un genrre, so es Maglam, duch de Albania, retingue Leir ab .lx. chavallers. E com fossen passats dos

5 Regularizo la ortografía y la acentuación, según las normas actuales de la Real Academia Española-Asociación de Academias de la Lengua Española (2010).

6 Se documenta también la variante "lx" en $F V$ (p. 25, nota 72).

7 En FV aparece “.xxx.”.

8 Suprimido en $G E$. 
anys e Leir continuament aturas e stigues ab son gendre, Goronilla, filla sua, enuja-sse'n per la multitud dels chavallers, qui desonraven los oficials e ministres de casa del duch d'Albania, com no eren mils e pus habundadament proveitz. E dix Goronilla a son marit lo duch que ordanas e manas a Leir, pare seu, que assats era que agues .xxx. chavallers en son servir e que lexas los altres .xxx. que havia (HRBC, p. 199).

Hallamos también ampliaciones (amplificatio) o alteraciones en el orden del discurso o de algunos términos:

Post hunc reganavit filius suus Rud Hudibras xxxix annis. Ipse populum ex civili discidio in concordiam reducens, condidit Kaerreint, id est Kantuariam. Condidit etiam Kaergueint, id est Guintoniam, atque oppidum montis Paladur, quod nunc Sephtonia dicitur. Ibi tunc aquila locuta est, dum murus aedificaretur, cujus sermones, si veros esse arbitrarer, sicut cetera memoriae dare non diffugerem (HRB, cap. 29).

Después d'este rey Leoír reinó un su fijo a que llamaron por nombre Rudohubras, y éste reinó de otra guisa que non su padre, y d'esto diremos adelante en su logar [...]; y después del rey Leír Rud Hubras su fijo. Otrosí diximos ante d'esto de cómo el rey Leír, padre del rey Rud, governara flacamente el reino yl perdiera por ello. Y empós esto aquel rey Rud su fijo que dize aquí la Istoria que reinó empós él pues que se fue bien apoderando del reino fizo a los pueblos y a los ricos ombres que se abiniesen, y sacó de entre ellos desabenencia y peleas, y partiólos de las asonadas con que avién confondido el reino, y fízolos aver paz y amor entre sí. Este rey Rud empós esto pobló una cibdad a que puso nombre Laerquem. Y según lo espone aquí esa istoria misma Laerquem es la cibdad Cantuaria. Y después de eso pobló otrosí otra cibdad, y llamóla Laerguenid, y ésta es a la que dixeron después Quintonia. Y pobló otrosí el castillo del monte Paladur, a que dixeron después Sephonia, y la cibdad d'este castiello fue cercada de muro y bien fuerte. Y cuenta la istoria que cuando fazié el muro d'esta cibdad que fabló ý una águila, mas peró non pone ý qué dixo aquella águila (GE, III/I, pp. 479-480, 499).

Apres de Leir regna lo seu fill Rudhudibras .xxx. anys, e com fos discordia en la terra, ell acorda.ls e adificha Kaerkam, ço es Contubera. Edifica, axi meteis, Caergem, ço es Guintonia, e.l castell del mont Paladur, qui ara es dit Sephonia. Aquí, dementre lo mur se edificava, una aguila comença de parlar, les paraules de la qual, si.m penssas que fossen veres, no tardaria de fer-ne memoria, axi com de les altres coses (HRBC, p. 197).

Ahora bien, del análisis detallado del léxico se desprende que hay cuatro casos relevantes que revisten especial interés y sobre los que debe fijarse la atención. El primero es el sustantivo "nepti" -'neptis' en nominativo- ("nupsit cuidam nepti Laviniae", $H R B$, cap. 6), que se vierte como "sobrina" ("sobrina de aquella reina Lavinia", $G E, \mathrm{iI} / 2$, p. 497), "neboda" ("una neboda de Lavinie", $H R B C$, p. I8I) y "nieta" ("una nieta de Laviniea", GCE, fol. 6 $\mathrm{I}^{\mathrm{r}-\mathrm{b}}$ ). Ambos significados se documen- 
tan en latín medieval ${ }^{9}$, pero lo que resulta destacable es que en la $G C E$ se emplee la forma "nieta"; ya que, si se aceptase que Fernández de Heredia trabajó a partir del texto catalán, como se ha sostenido, se plantea una pregunta inevitable: por qué no lo vertió como "sobrina", tal y como se utiliza en esa versión ("neboda"). A este respecto, podrían proponerse dos posibles explicaciones: o bien, un copista habría cambiado "néta" por "neboda" en el proceso de transmisión textual, y Heredia habría dispuesto de una copia distinta a la conservada; o bien, el autor aragonés había trabajado a partir de un texto original en latín, al igual que la $H R B C$ o la $G E$, donde se indica explícitamente ("e aquellas palavras de la oración de Bruto son en aquella estoria de Bretaña en latín, como todas las otras razones d'ese libro", $G E$, II/2, p. 5II), y no de una versión intermedia en catalán.

El segundo caso tiene que ver con el vocabulario militar y ético. El sintagma "militia et probitate" ("In tantum autem militia et probitate vigere coepit", $H R B$, cap. 7), traducido como "bondat" y "por valer cavallería e por otras bondades" (“ e tanto aprovó allí en bondat e començo a valer por cavallerías e por otras bondades", $G E, \mathrm{II} / 2$, p. 498), "prous e valent" y "en chavalleria e en tota proesa" ("e comenssa a esser axi prous e valent en chavalleria e en tota proesa", $H R B C$, p. I82) y "ardit et valient" y "en cavallerías et en todas virtudes" "e començó seyer assí ardit et valient en cavallerías et en todas virtudes", $G C E$, fol. $\left.6 \mathrm{I}^{\mathrm{v}-\mathrm{a}}\right)$, pone de manifiesto que las tres versiones ofrecen para ambos sustantivos sus respectivas formas consolidadas en romance ("militia" identificada con "cavallería", "chavalleria" y "cavallerías", y "probitate" con "bondat" - con el sentido de capacidad en el arte de la guerra, "prous e valent" y "ardit et valient") y que, dada la precisión léxica, se puede inferir que sus traductores eran conocedores del ámbito caballeresco o pertenecían a él.

El tercer caso, como el precedente, se refiere también al vocabulario militar. Se trata del sustantivo "milites" y del sintagma "campestre praelium" ("quia tot milites non habebant quot sibi ad campestre praelium committendum sufficerent, $H R B$, cap. II; y en variante "exercitum" en $F V$, cap. 7), vertidos como "cavalleros" y "batalla campal" ("porque non avié tantos cavalleros cuantos le cumpliesen para salir a venir a ellos a darles batalla campal", $G E, \mathrm{II} / 2$, p. 503), "cavallers" y "batalla campal" ("com no havia tants chavallers que li bastas a fer batalla campal", $H B R C$, p. I84) y "cavalleros" y "batalla campal" ("porque no havía tantos cavalleros que le bastassen assaz la batalla campal", $G C E$, fol. $63^{\mathrm{r}-\mathrm{b}}$ ), que, de nuevo, muestran que se siguen los usos habituales en romance y que remiten a un entorno en contacto con el ámbito caballeresco.

9 Agradezco al Dr. José Martínez Gázquez (Institut d’Estudis Medievals, Universitat Autònoma de Barcelona) las aclaraciones e indicaciones facilitadas a este respecto. 
Y, el cuarto y último caso es la cifra "CCCXIv" ("Quae, ut collectae fuerunt, cCCxiv numero", $H R B$, cap.I5; y en variantes "trecente .xximII." en $V F$, cap. I5 y "CCC.XX.III" en BernM, cap. I5), que aparece como "trezientas e veinte e cuatro" ("e ayuntáronlas luego, e fueron por cuenta trezientas e veinte e cuatro", $G E$, II/2, p. 5IO), "CCCXXIIII" ("e com fossen totes ajustades, foren .CCCXXIIII. per comte", $H R B C$, p. I87) y "CCC.XX.IIII." ("Et como fuessen todas aplegadas .CCC.XX.III por conto", $G C E$, fol. $\left.65^{\mathrm{v}-\mathrm{a}}\right)$, que conduce a pensar que la fuente o fuentes a la(s) que tuvieron acceso los traductores de la versión en castellano, en catalán y en aragonés debían pertenecer al corpus de manuscritos que contienen variantes textuales.

De los datos anteriores, a pesar de su escaso número, es posible concluir, por un lado, que los traductores de estas tres versiones hispánicas conocían el mundo de la caballería y de su ética; y, por el otro, que para realizar su labor debieron utilizar manuscritos que contenían variantes textuales respecto del considerado texto 'canónico', por lo que es dable señalar que un análisis que recogiese todas las variantes más significativas y su contraste con cada uno de los textos hispánicos, labor que excede los límites de este trabajo, podría contribuir a aclarar qué versión o versiones de la $H R B$ pudieron circular por la Península Ibérica y a obtener, de ese modo, un mejor conocimiento de la recepción y difusión de la Materia de Bretaña en los tierras hispánicas.

\section{BIBLIOGRAFÍA}

Alfonso X el Sabio, 2009: General Estoria (ed. P. Sánchez-Prieto Borja), Io vols, Madrid.

Besamusca, B., 2000: "The Medieval Dutch Arthurian Material", en W. H. Jackson y S. A. Ranawake (eds.), The Arthur of the Germans. The Arthurian Legend in Medieval German and Dutch Literature, Cardiff, pp. 187-228.

Bohigas, P., I985: "Història desl reis de Bretanya des de Brut fins a Dunvallo. Traducció catalana de la 'Historia Regum Britanniae' de Galfred de Monmouth. Llib. I”, en Sobre manuscrits biblioteques, Barcelona, pp. 178-203.

Cacho Blecua, J. M., 1997: El gran maestre Juan Fernández de Heredia, Zaragoza. Echard, S., 20II: "Geoffrey of Monmouth", en S. Echard (ed.), The Arthur of Medieval Latin Literature. The Development and Dissemination of the Arthurian Legend in Medieval Latin, Cardiff, pp. 45-66.

Faral, E., 1993: La légende arthurienne. Études et documents, 3 vols, Paris.

Fernández de Heredia, J.: La Grant Crónica de Espanya (Ms. I0.I33 BNE, Madrid, $<$ http://www.bne.es/es/Catalogos/BibliotecaDigital>. 
Geoffrey de Monmouth, 1984: Historia de los reyes de Bretaña (ed. L. A. de Cuenca), Madrid.

Gropper, S., 20II: "Breta Sögur and Merlinuisppa", en M. Kalinke (ed.), The Arthur of the North. The Arthurian Legend in the Norse and Rus' Realms, Cardiff, pp. 48-6o.

Kasten, Ll., 1970: "The Utilizzation of the Historia Regum Britanniae by Alfonso X”, Hispanic Review, 38/5, pp. 97-II4.

Real Academia Española-Asociación de Academias de la Lengua Española, 20ıо: Ortografía de la lengua española, Madrid.

Rico, F., I984: Alfonso el Sabio y la "General estoria", Barcelona.

Wright, N., 1985-1991: The Historia Regum Britanniae of Geoffrey of Monmouth, 5 vols, Cambridge.

- 1985: The Historia Regum Britanniae of Geoffrey of Monmouth. Bern Burgerbibliothek MS. 568 , Cambridge, vol. I.

Wright, N., 1988: The Historia Regum Britanniae of Geoffrey of Monmouth. The First Variant Version: a critical edition, Cambridge, vol. II. 
